\title{
Organização da atenção odontológica no município: estudo de caso
}

\author{
Municipal organization of dental services: case study
}

Organización de la atención dental en la ciudad: estudio de caso

\author{
Suzely Adas Saliba MOIMAZ ${ }^{1}$ \\ Audrey Quintella Coelho OKAMURA ${ }^{2}$ \\ João Guilherme Rodrigues NAYME \\ Lenise Patrocinio Pires CECILIO ${ }^{3}$ \\ Cléa Adas Saliba GARBIN ${ }^{1}$ \\ Tânia Adas SALIBA ${ }^{4}$ \\ Nemre Adas SALIBA ${ }^{\mathbf{1}}$
}

${ }^{1}$ Professora Titular do Departamento de Odontologia Infantil e Social,

Faculdade de Odontologia de Araçatuba, UNESP Univ. Estadual Paulista 16015-490 Araçatuba-SP, Brasil

${ }_{2}^{2}$ Mestranda, Programa de Pós-Graduação em Odontologia Preventiva e Social da Faculdade de Odontologia de Araçatuba, UNESP Univ. Estadual Paulista 16015-490 Araçatuba-SP, Brasil

${ }^{3}$ Mestre, Programa de Pós-Graduação em Odontologia Preventiva e Social da Faculdade de Odontologia de Araçatuba, UNESP

Univ. Estadual Paulista 16015-490 Araçatuba-SP, Brasil

${ }^{4}$ Professora Adjunto do Departamento de Odontologia Infantil e Social,

Faculdade de Odontologia de Araçatuba, UNESP Univ. Estadual Paulista 16015-490 Araçatuba-SP, Brasil

\begin{abstract}
Resumo
Dentre os maiores desafios da administração pública está a organização do serviço de saúde. Exemplos de administração na área odontológica subsidiam gestores, tanto no planejamento, quanto na execução do trabalho. O objetivo desse estudo foi descrever a organização do serviço de saúde bucal municipal. Trata-se de um estudo exploratório e descritivo, realizado em município do estado São Paulo. Foram definidas as categorias: características sócio-demográficas do município; configuração da política de saúde bucal e sua relação com as diretrizes nacionais; descrição da infraestrutura física e recursos humanos da rede de serviços odontológicos; organização do cuidado nos níveis de atenção. Foi realizada análise de banco de dados secundários e entrevista semiestruturada com a Coordenadora de Saúde Bucal. O município possuía 10 Unidades Básicas de Saúde, com 13 Equipes de Saúde da Família (ESF), 5 delas com Equipes de Saúde Bucal. A Atenção Básica contava com 10 Cirurgiões-Dentistas sendo 5 da ESF; 25 Auxiliares em Saúde Bucal e 5 Técnicos de Saúde Bucal. Havia um Centro de Especialidades Odontológicas com 5 especialistas. Os Agentes Comunitários de Saúde faziam o cadastramento das famílias, classificadas conforme critérios de risco. Os níveis de atenção à saúde bucal estavam integrados e a demanda organizada, permitindo que os serviços odontológicos fossem oferecidos de maneira equânime à população.

Descritores: Sistema Único de Saúde; Saúde Bucal; Gestão em Saúde; Organização e Administração; Atenção Primária à Saúde.
\end{abstract}

\begin{abstract}
Among the greatest challenges of public administration is the organization of the health service. The examples of administration in the dental area subsidize managers, both in planning and execution of work. The aim of this study was to analyze the municipal organization of dental services. It's an exploratory and descriptive study with qualitative profile. The following categories were defined: local socio-demographic characteristics, oral health policy and its relation to national guidelines, description of the physical infrastructure and human resources of the dental services network, organization and integration between primary and specialized health care. Data were collected trough a review, documental analysis, and analysis of secondary data and semi-structured interview with the Municipal Coordinator of Oral Health. It had 10 Basic Health Unities with 13 Family Health Teams, 5 of them with Oral Health Teams. Basic Care with 10 dentists, of which 5 belong to the Family Health Strategy; 25 Dental Auxiliaries and 5 Hygienists. There was a Dental Specialist Center with 5 experts. The Community Health Agents registered the families, classifying them according to risk criteria. The levels of oral health care were integrated and the demand was organized, allowing dental services to be offered equitably to the population.

Descriptors: Unified Health System; Oral Health; Health Management; Organization and Administration; Primary Health Care.
\end{abstract}

\section{Resumen}

Entre los mayores desafíos de la administración pública está la organización del servicio de salud. Los ejemplos de administración en los servicios dentales subsidian a los administradores, tanto en la planificación como en la ejecución del trabajo. El objetivo de este estudio fue analizar la organización de los servicios dentales de una ciudad. Se trata de un estudio exploratorio y descriptivo de perfil cualitativo. Se definieron las siguientes categorías: características socio-demográficas locales, la política de salud oral y su relación con las directrices nacionales, descripción de la infraestructura física y los recursos humanos de la red de servicios dentales, organización e integración entre la atención primaria y especializada. Los datos se recogieron a través de una revisión, análisis de documentos y análisis de datos secundarios; entrevista semiestructurada con el Coordinador Municipal de la salud oral. Tenía 10 Unidades Básicas de Salud con 13 equipos de Salud Familiar, 5 de ellos con Equipos de Salud Bucal. Atención básica con 10 dentistas, de los cuales 5 pertenecen a la Estrategia de Salud de la Familia; 25 Auxiliares Dentales y 5 Higienistas. Había un Centro de Especialistas Dentales con 5 expertos. Los Agentes Comunitarios de Salud han registrado a las familias, clasificándolas según criterios de riesgo. Los niveles de atención de la salud oral se integraron y se organizó la demanda, permitiendo que los servicios dentales se ofrecieran de manera equitativa a la población.

Descriptores: Sistema Único de Salud; Salud Bucal; Gestión en Salud; Organización y Administración; Atención Primaria de Salud.

\section{INTRODUÇÃO}

O último levantamento epidemiológico nacional em saúde bucal, SB Brasil 2010 ${ }^{1}$, indicou disparidades sociais na distribuição das doenças bucais, sinalizando desigualdades no acesso ao atendimento básico de populações com maior risco social. Esse dado mostrou que, ainda hoje, a organização do serviço de maneira universal, 
integral e resolutiva é um dos principais nós na gestão ${ }^{2}$. As políticas públicas devem buscar de maneira contínua e por meio de ações articuladas em redes, promover a coordenação do cuidado entre os níveis de atenção. Porém muitos serviços ainda atuam de forma fragmentada, apesar dos grandes avanços nos últimos anos ${ }^{3}$.

Em especial nos serviços de odontologia, comumente pouco integrados à atenção básica, direcionados a práticas curativas e com problemas de alta prevalência na população, existem vários desafios na organização do sistema público de saúde. Portanto, é necessário dominar os principais recursos para tal tarefa, como a elaboração de planos, a verificação e controle das ações, tal como a vigilância dos indicadores de saúde bucal ${ }^{4}$.

Embora haja atendimento público há vários anos no Brasil, esse esteve direcionado apenas aos escolares. Mesmo assim, o país foi apontado como um dos campeões mundiais da cárie dentária em 1986, quando atingiu CPO-D médio de 6,65 aos 12 anos, considerado de alta prevalência ${ }^{5}$. Somente com a introdução da Política Nacional de Saúde Bucal e atendendo ao princípio de universalidade do Sistema Único de Saúde (SUS), a atenção foi garantida a todas as faixas etárias ${ }^{2}$.

As políticas públicas direcionadas à saúde bucal ganharam espaço com a inserção das Equipes de Saúde Bucal (ESB) na Estratégia de Saúde da Família (ESF) ${ }^{6,7}$. Formuladas especificamente para a área, reúnem uma série de ações voltadas para a população, baseadas nos princípios de universalidade, equidade e integralidade. Visam a reorganização dos serviços públicos ofertados em todos os níveis de atenção, independente da localidade na qual serão desenvolvidos ${ }^{6,8}$.

A fim de garantir a integralidade nas ações, instituíram-se os Centros de Especialidades Odontológicas (CEO) e os Laboratórios Regionais de Próteses Dentárias (LRPD). Um dos objetivos é ampliar o acesso aos tratamentos mais complexos em saúde bucal, enquanto a atenção básica deve atender a maior parcela dos problemas que a população demanda, de forma a aumentar a resolubilidade e referenciar o menor número possível de casos para a atenção especializada ${ }^{3}$.

Dentre as ferramentas disponíveis ao gestor encontrase o protocolo de avaliação diagnóstica da saúde bucal na atenção básica. Identificam-se os diferentes grupos sociais e suas características peculiares, as necessidades são hierarquizadas e assim é possível organizar o atendimento numa agenda, abrangendo assim a unidade de saúde, a família e a comunidade, eixos de atuação da Estratégia de Saúde da Família 9 .

Nesse contexto, o relato de experiências bem sucedidas pode subsidiar gestores na elaboração, adequação e mudança na organização dos serviços sob sua responsabilidade.

Desta forma, o objetivo neste estudo foi analisar os serviços de saúde bucal de um município do estado de São Paulo, com enfoque na organização da demanda.

\section{MATERIAL E MÉTODO}

Trata-se de um estudo exploratório e descritivo dos serviços de saúde bucal de Penápolis, região noroeste do estado de São Paulo. A escolha desse município teve como critérios o serviço estruturado nos três níveis de atenção e a possibilidade de acesso aos dados.

Foram utilizadas as seguintes estratégias metodológicas: revisão bibliográfica, análise documental, análise de bases de dados secundários e entrevista semiestruturada com a Coordenadora de Saúde Bucal, a fim de obter informações sobre as características de funcionamento e organização específicas do município.

A análise documental incluiu a legislação e as publicações disponíveis no sítio do Ministério da Saúde ${ }^{6,10,11,12,13}$.

Com base no referencial teórico-metodológico, foram previamente definidas e ajustadas as seguintes categorias de análise:

- Características sociodemográficas do município;

- Configuração da política de saúde bucal local e sua relação com as diretrizes nacionais;

- Descrição da infraestrutura física e recursos humanos da rede de serviços odontológicos;

- Organização do cuidado nos níveis de atenção. Diz respeito à relação entre as Unidades Básicas de Saúde (UBSs) e o Centro de Especialidades Odontológicas (CEO), abrangendo regras de funcionamento e organização, tipos de ações oferecidas, fluxos dos serviços, por meio da análise dos mecanismos de referência e contra-referência e do agendamento para atendimento especializado e organização da demanda.

O projeto foi submetido e aprovado pelo Comitê de Ética em Pesquisa da Faculdade de Odontologia de Araçatuba - UNESP (Processo FOA - 01025/2011), respeitando-se os ditames éticos da Resolução 466/12.

Para a realização da pesquisa, houve a aprovação do secretário municipal de saúde e a obtenção de autorização para a execução da entrevista e coleta de dados.

\section{RESULTADOS E DISCUSSÃO}

- Características sociodemográficas do município

O município de Penápolis está localizado no noroeste do Estado de São Paulo, com 58.510 habitantes (Censo 2010). A população vive predominantemente na área urbana $(95,5 \%)$, o que proporciona acesso facilitado aos serviços de saúde e contribui para a captação de pacientes e execução de ações coletivas de educação em saúde. Os gastos com saúde correspondem a $22,79 \%$ da receita arrecadada, segundo último relatório divulgado pelo Ministério da Saúde, enquanto o percentual mínimo, de acordo com a emenda constitucional 29, é de 15\% (CONOF/CD, 2012).

O município tem como base econômica a agroindústria canavieira, a pecuária e lavouras de café. Possui um distrito industrial e um comércio diversificado. A indústria canavieira desde alguns anos tem se expandido a ponto de se constatar que a agricultura familiar e bacia leiteira não são mais praticadas. Entretanto, há uma crise severa na área, prejudicando milhares de trabalhadores. Ademais, esse perfil econômico confere ao município uma variação populacional relacionada à sazonalidade da safra da cana, com migração de um grupo de trabalhadores rurais advindos de outras regiões brasileiras. Como consequência, formou-se um grupo de grande vulnerabilidade social, com necessidades acumuladas em saúde, condições precárias de trabalho e moradia, e riscos sociais relacionados ao uso de álcool, drogas e prostituição. Essas características locais são importantes para o planejamento das ações em saúde, o qual deve ser pautado na realidade da população, direcionando e adequando as ações preventivas e curativas aos que mais necessitam e buscam por atendimento.

- Configuração da política de saúde bucal e sua relação com as diretrizes nacionais

A atenção em saúde bucal no município evoluiu conforme o restante do país, tendo partido do excludente 
sistema incremental e caminhado um longo caminho até a implementação da Política Nacional de Saúde Bucal. A população adulta e idosa acessava apenas o serviço de urgência em que eram realizadas principalmente extrações dentárias. Mesmo a parcela assistida, crianças e adolescentes até 14 anos de idade, recebia apenas aplicações tópicas de flúor e tratamentos de baixa complexidade.

Desde então, muito foi conquistado para a comunidade do município no âmbito da saúde, pois a mesma tem sido reconhecida como prioridade na gestão. Ali se encontra a sede do primeiro Consórcio Intermunicipal de Saúde criado no Brasil, em 1986, a qual é formada atualmente por 7 municípios. Foi pioneiro nas ações de municipalização propostas pela Lei $\mathrm{N}^{\circ} 8.080 / 90^{14}$, assim como nas mudanças trazidas pelas diretrizes propostas pelo SUS.

O serviço de saúde foi descentralizado gradativamente, com a abertura de unidades de saúde e implantação da Estratégia Saúde da Família (ESF). Já a atenção odontológica manteve-se em um espaço único para atenção básica e, a partir de 2008, também para a atenção especializada, característica frequente em pequenos municípios. A centralização do serviço odontológico, independente do nível de atenção, influenciou vários aspectos como o acesso da população adstrita da ESB na ESF, agendamento de pacientes, integração entre as equipes, produção, cumprimento de metas e sistema de referência e contra-referência ${ }^{15}$.

Em diversos municípios, a prestação dos serviços de saúde é configurada em redes de atendimento e as referências da atenção básica a outros níveis de atenção não oferecidos localmente seguem as normativas pactuadas com municípios de maior porte. As redes de serviço não são apenas um padrão de interação, mas a forma pela qual o serviço público é "planejado, concebido, produzido e oferecido à população" "16. O processo de pactuação é mais que a interação entre as instâncias de poder ou entre órgãos geograficamente dispersos, sendo concebido como "uma linguagem de vínculos das relações sociais entre as organizações" "17. A questão é como convencer os diferentes atores das diferentes organizações a falarem a mesma "linguagem", suficientemente eficaz para gerar "vínculos"18.

A avaliação de qualidade dos serviços odontológicos prestados era realizada por meio de métodos desenvolvidos pelo próprio município, dentre eles destacaram-se pesquisas com usuários, relatórios de ouvidoria e indicadores de produtividade. Além disso, avaliou-se o cumprimento de metas estabelecidas pelo governo federal. Mesmo considerando que a centralização das ações de odontologia não seja indicada, os resultados obtidos nos processos de avaliação sempre foram positivos. Ainda assim, nos últimos anos os serviços odontológicos do município foram reconfigurados através da descentralização no sentido dos bairros, as Unidades Básicas de Saúde. Foram observadas as recomendações ministeriais, as formas de organização sugeridas para o serviço público e considerando o crescimento da cidade, de forma a garantir acessibilidade aos residentes de áreas mais periféricas.

Segundo Neto et al. ${ }^{19}$, o modelo de gestão descentralizada faz parte do processo de construção das perspectivas dos serviços de saúde por envolver um conjunto de atores locais e regionais para uma nova organização dos serviços.

O município apresentava uma clínica odontológica central onde trabalhavam as 5 ESB tradicionais e o CEO, além de 2 equipes da ESF abrigadas temporariamente. Outras 3 ESB da ESF estavam descentralizadas, atuando junto com os demais membros da estratégia, com objetivo de ampliar o acesso aos serviços de saúde, conforme estabelecido pelas diretrizes do SUS.

\section{- Descrição da infraestrutura física e recursos humanos da rede de serviços odontológicos}

O município possuía 13 Equipes de Saúde da Família, atuando em 10 Unidades Básicas de Saúde, 5 delas com ESB da ESF.

A Atenção Básica Odontológica possuía, ao todo, 10 Cirurgiões-Dentistas (CD), os quais atendiam a demanda das 10 UBSs do município. Cada um deles era responsável pela população adstrita do território da unidade de saúde ao qual estava cadastrado. Apenas 5 equipes estavam cadastradas como ESB da ESF, porém todas trabalhavam seguindo os princípios de territorialização, vínculo e adscrição de clientela. Esse é um aspecto importante, pois auxilia no acompanhamento de rotina e na busca da satisfação das necessidades de saúde da população ${ }^{19}$.

A configuração numérica das equipes de saúde bucal da ESF foi determinada por gestões anteriores. Como preconizado pela política nacional em $2001^{6}$, para cada duas equipes da estratégia cadastrou-se uma equipe de saúde bucal. Adequar os profissionais da atenção tradicional para seguirem a lógica praticada pela ESF favoreceu o vínculo e a integração da população ao serviço. A comunidade sentiu-se melhor cuidada, pois a equipe tinha uma visão ampliada do território e das necessidades dos usuários. Assim foi possível estimular a autonomia e a participação no tratamento, numa relação de respeito e valorização das particularidades, inclusive corresponsabilizando a população pelo seu próprio bem-estar $^{19,20}$.

O município possuía 6 equipes cadastradas no ministério desde 2004. Até o momento 5 haviam sido implantadas. A sexta equipe aguardava readequação física de uma UBS para iniciar suas atividades, portanto espera-se que a cobertura dos serviços oferecidos à população aumente em breve. É interessante observar que a cobertura da atenção básica era de $67 \%$ da população enquanto apenas 33\% era realizada pelas equipes da ESF.

Além das 10 equipes de atenção básica, existia um CEO com 5 especialistas. Os CDs trabalhavam em conjunto com 25 Auxiliares em Saúde Bucal (ASBs), 5 Técnicos em Saúde Bucal (TSBs), 2 assistentes administrativos e 1 auxiliar de serviços gerais.

A unidade de atendimento central possuía 11 consultórios odontológicos completos, 5 com aparelhos de radiografias bucais. Existiam consultórios odontológicos em outras três UBSs, nas quais atuavam 2 equipes da ESF do tipo 2 ( $1 \mathrm{CD}, 1 \mathrm{TSB}$ e $2 \mathrm{ASB})$ e 1 equipe do tipo 1 ( $1 \mathrm{CD}$ e 1 ASB). Uma nova unidade com 3 consultórios estava em fase de implantação abrigará uma equipe de cada tipo. Essas equipes estavam em atuação, de maneira provisória no Centro Odontológico. Além disso, existia 1 consultório localizado no Ambulatório de DST/HIV/AIDS e outro na Associação de Pais e Amigos dos Excepcionais (APAE), voltado para pacientes portadores de necessidades especiais, totalizando 18 consultórios implantados.

$\mathrm{O}$ acesso era universal e para a organização da demanda da atenção básica, as famílias eram cadastradas em suas residências pelos agentes comunitários de saúde. Era preenchida uma ficha odontológica específica do serviço, com informações sobre variáveis econômicas, sociais e agravos de saúde. Incluía informações sobre necessidades odontológicas referidas, última visita ao dentista e uso de próteses dentárias. A classificação econômica era realizada 
por meio do Critério de Classificação Econômica Brasil. Gerava-se um escore de A a E, sendo que E representa menor poder de compra ${ }^{21}$. A priorização das famílias, dentro de cada faixa econômica, era realizada de acordo com as variáveis: queixa referida, família com ou sem criança e presença de agravo, gerando escores de classificação que variavam de 1 a 8 , para cada faixa econômica, como mostrado na Figura 1.

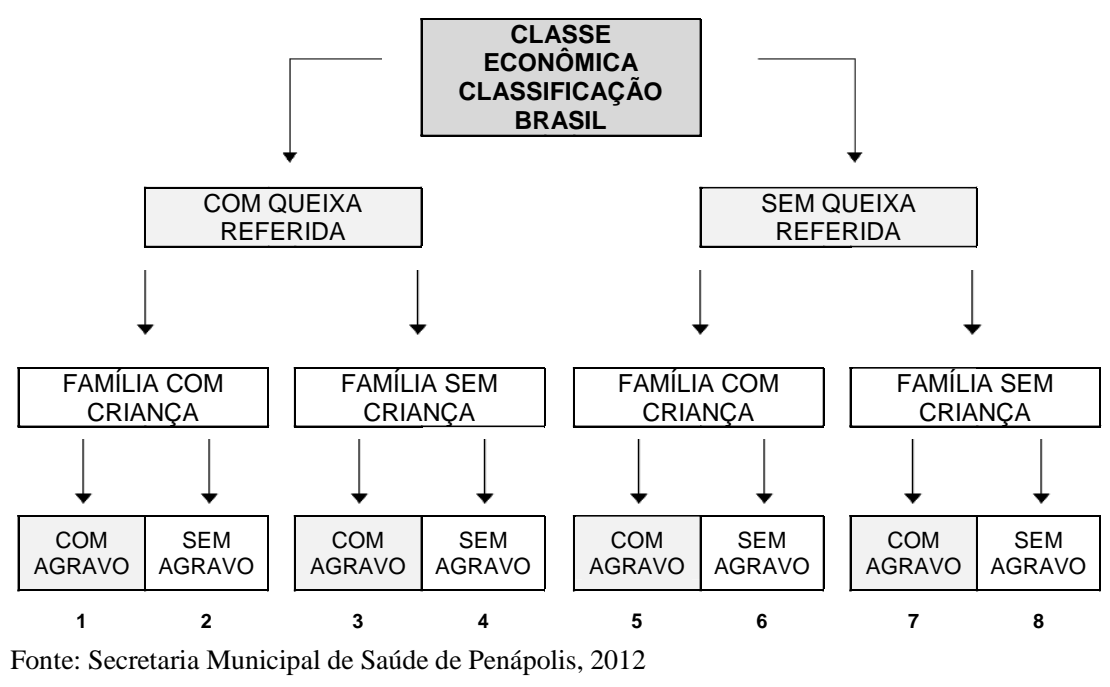

Figura 1: Priorização das famílias, dentro de cada faixa econômica, de acordo com as variáveis: queixa referida, família com ou sem criança e presença de agravo.

O agendamento era feito de acordo com os escores, priorizando as famílias classificadas como E1. O convite era entregue pelos Agentes Comunitários de Saúde em cada residência, contendo todas as informações necessárias para dar início ao tratamento.

Após a consulta inicial, era feita a classificação de risco à cárie, doença periodontal e tecidos moles, de acordo com os graus, em baixo risco, risco moderado e alto risco, de acordo com as diretrizes de saúde bucal estabelecidas pela "Secretaria de Saúde do Município de São Paulo",22. Prioridades clínicas foram estabelecidas para o atendimento a fim de organizar a agenda de trabalho. Este sistema possibilitou a busca ativa das famílias mais vulneráveis, eliminando as filas em dias de agendamento, e o atendimento às necessidades de saúde bucal da população baseadas em risco social e clínico. Assim como o estudo desenvolvido por Alves ${ }^{23}$, o emprego da abordagem sóciodental possibilitou sistematizar de forma eficiente, informações relevantes para a organização da atenção em saúde bucal nas ESF a partir da compreensão sobre a relação entre condições de vida das famílias e medidas clínicas bucais. $\mathrm{O}$ mesmo autor $^{23}$ afirmou que a combinação de medidas normativas em saúde bucal, associadas a medidas sobre condições de vida, mostraram-se ferramentas importantes para a redução das principais dificuldades encontradas pelas ESBs na Estratégia, tais como melhor organização da demanda e definição de indivíduos com prioridade de cuidado, influenciando a resolutividade do sistema.

Através de convite entregue nas próprias residências, aproximadamente 25 famílias a cada mês, ou 100 pessoas, tem sido chamadas para consulta odontológica por ESB. Observou-se que parte dos indivíduos não comparecem, mas as razões permanecem desconhecidas. Havia políticas públicas para atendimento de pacientes especiais, gestantes, crianças e adolescentes, renais crônicos, diabéticos, hipertensos e ações de promoção e educação em saúde bucal, realizados em diferentes espaços sociais e públicos do município, eventos, escolas, empresas. Essas ações podem melhorar a adesão das famílias ao tratamento odontológico, segundo diversos estudos ${ }^{24,25}$.
O programa de saúde bucal direcionado às gestantes fazia parte do pré-natal. O encaminhamento era realizado pelas UBSs assim que a gestação era identificada. Quando não eram acompanhadas pelo serviço público e sim por médico particular, podia-se realizar o pré-natal odontológico caso houvesse interesse.

A demanda para atendimento de crianças de 0 a 14 anos era organizada durante a classificação de prioridade das famílias. Se esses indivíduos buscassem o serviço odontológico, mesmo antes de a família ter sido selecionada, haveria o cadastramento e agendamento desses pacientes, independente da condição familiar, por serem considerados como população prioritária.

O encaminhamento de diabéticos e hipertensos tem sido realizado pelas equipes médicas das UBSs, porém os programas direcionados a esses pacientes estavam em fase de implementação.

A população contava com serviço de urgência e emergência diário. Tratava-se de pronto atendimento para qualquer queixa referida, de acesso aberto e ilimitado no horário de funcionamento do estabelecimento. Em dois dias da semana operava em regime ampliado até às 21 horas, a fim de possibilitar o acesso da população que não poderia frequentar o serviço durante o dia. Nesse ambiente, muitos pacientes eram triados e colocados em tratamento por risco clínico, ou encaminhadas à atenção secundária, conforme a necessidade.

Além da busca espontânea, os indivíduos poderiam ter suas necessidades detectadas durante as atividades educacionais realizadas nos espaços sociais e escolas do município e então encaminhados pela ASB para a clínica.

\section{- Organização do cuidado nos níveis de atenção}

O acesso aos serviços odontológicos da atenção básica era feito pela classificação de risco, a qual é conhecida pela população. Ressalta-se que havia participação dos usuários do serviço nas decisões e reuniões para discussão e definição dos critérios. Os principais problemas e queixas, bem como sugestões para melhoria na organização da demanda, foram levantados junto aos usuários do sistema e funcionários, por meio da realização de grupos de trabalho e rodas de discussão. Uma queixa frequente era que muitas pessoas carentes precisavam de tratamento e não o conseguiam, o que culminou na adoção da Classificação Brasil como um critério de priorização. Os profissionais enfatizaram as necessidades clínicas e de risco social, que determinaram a inserção da queixa referida e dos agravos de saúde no protocolo de agendamento e encaminhamento de pacientes, estabelecido pelo serviço odontológico do município $^{15,26}$.

Para os gestores, a maior preocupação era com o sistema de referência e contra-referência que, se organizado de maneira adequada, evita desperdício de recursos e aumenta a produtividade da equipe, culminando no incremento da resolutividade. A somatória dos aspectos apontados como determinantes pelos usuários, trabalhadores e gestores, possibilitou a construção do atual protocolo de trabalho. O encaminhamento ao Centro de Especialidades Odontológicas era feito pela atenção básica quando havia necessidade de tratamento especializado. Era realizado por meio de fichas desenvolvidas pelo próprio serviço, entregues aos critérios de prioridade, podendo o próprio paciente fazêlo na recepção do estabelecimento, incluindo em período noturno. Não se observou demanda reprimida para nenhuma especialidade e o agendamento da primeira consulta não ultrapassava 15 dias de espera. 
O Centro de Especialidades Odontológicas do município era do tipo II, por apresentar quatro ou mais cadeiras $^{13} \mathrm{e}$ atendia às especialidades de endodontia, periodontia, cirurgia oral menor, semiologia e pacientes especiais.

Os Cirurgiões-Dentistas das UBSs do município seguiam os critérios estabelecidos pelo Ministério da Saúde para referenciar os pacientes para os $\mathrm{CEOs}^{27}$. Somente a periodontia realizava procedimentos não adequados à especialidade. Mesmo que boa parte dos problemas pudesse ser resolvida na atenção primária, o encaminhamento ao CEO servia de escape para a maior demanda do município. Quando a atenção básica ficava sobrecarregada de pacientes com problemas periodontais, o atendimento das demais necessidades da população acaba comprometido entre os níveis de atenção, segundo diversos autores ${ }^{8,28}$. Essa é uma das questões mais críticas na organização dos serviços de saúde bucal, entretanto pode ser solucionada com a contratação e capacitação de profissionais que referenciam de maneira adequada os procedimentos que necessitem de atenção especializada, inclusive dos Agentes Comunitários de Saúde ${ }^{29}$. Além disso, a adoção de uma agenda de saúde desenvolvida de acordo com as necessidades específicas do município é indicada para a organização da demanda ${ }^{9}$.

O município atingiu as metas estabelecidas pelo Ministério da Saúde em quase todas as especialidades ofertadas, exceto na especialidade de cirurgia, provavelmente por exigir procedimentos muito complexos, os quais demandam maior tempo de atendimento e que não são comumente encontrados na população. Não havia demanda reprimida para tal especialidade e a inadequação quantitativa da meta não refletia a realidade da atenção, ou seja, o atendimento integral da demanda ${ }^{15}$.

Analisar a organização dos serviços de saúde, com a participação de toda a equipe envolvida e os usuários, é importante para a identificação dos nós. Dessa maneira é possível buscar melhorias em diversos aspectos, como aumento da produtividade e resolubilidade, organização da agenda de trabalho, redução dos gastos e melhor aproveitamento dos recursos humanos.

\section{CONCLUSÃO}

Os níveis da atenção à saúde bucal estão integrados e a demanda organizada de acordo com critérios de risco social e condições clínicas. Não há demanda reprimida na atenção especializada, entretanto, alguns procedimentos da atenção básica têm sido realizados nesse ambiente. Não foi possível determinar se o acesso tem sido na prática universal e equânime.

\section{REFERÊNCIAS}

1. Brasil. Ministério da Saúde. Secretaria de Atenção à Saúde. Departamento de Atenção Básica. Coordenação Nacional de Saúde Bucal. SB Brasil 2010: resultados principais. Brasília: MS, 2011.

2. Aquilante AG, Aciole GG. O cuidado em saúde bucal após a Política Nacional de Saúde Bucal - "Brasil Sorridente": um estudo de caso. Ciênc saúde coletiva. 2015; 20(1):239-48.

3. Figueiredo N, Goes PSA. Construção da atenção secundária em saúde bucal: um estudo sobre os Centros de Especialidades Odontológicas em Pernambuco, Brasil. Cad Saúde Publica. 2009; 25(2):259-67.

4. Lessa CFM, Vettore MV. Gestão da Atenção Básica em Saúde Bucal no Município de Fortaleza, Ceará, entre 1999 e 2006. Saúde Soc. 2010;19(3):547-56.
5. Brasil. Ministério da Saúde. Divisão Nacional de Saúde Bucal. Levantamento Epidemiológico em Saúde Bucal: Brasil, zona urbana. 1986. Brasília: Ministério da Saúde, 1988b. Série C, Estudos e Projetos, v.4. 137p.

6. Brasil. Ministério da Saúde. Programa Saúde da Família - PSF. Brasília: Ministério da Saúde, 2001.

7. Nascimento AC, Moyses ST, Bisinelli JC, Moyses SJ. Saúde bucal na estratégia de saúde da família: mudança de práticas ou diversionismo semântico. Rev Saúde Publica. 2009; 43(3):455-62.

8. Morris AJ, Burke FJT. Primary and secondary dental care: how ideal is the interface. Br Dent J. 2001; 191(12):666-70.

9. Moimaz SAS, Melo LMLL, Garbin CAS, Garbin AJI, Saliba NA. Oral health assessment protocol in primary care. RGO. 2015; 63(4):446-54.

10. Brasil. Ministério da Saúde. Portaria 599, de 23 de março de 2006. Diário Oficial da União, Brasília, 24 de março de 2006.

11. Brasil. Ministério da Saúde. Portaria 600, de 23 de março de 2006. Diário Oficial da União, Brasília, 24 de março de 2006.

12. Brasil. Ministério da Saúde. Brasil sorridente: a saúde bucal levada à sério. Brasília, 2010.

13. Brasil. Ministério da Saúde. Portaria 1341/GM, de 13 de junho de 2012.

14. Neves LA, Ribeiro JM. Consórcio de saúde: estudo de caso exitoso. Cad Saude Publica. 2006; 22(10):2207-17.

15. Saliba NA, Nayme JGR, Moimaz SAS, Cecilio LPP, Garbin CAS. Organização da demanda de um Centro de Especialidades Odontológicas. Rev Odontol UNESP. 2013; 42(5):317-23.

16. McGuire M. Managing networks: propositions on what managers do and why they do it. Public Adm Rev. 2002; 62(5):599-609.

17. Junqueira LAP. Intersetorialidade, transetorialidade e redes sociais na saúde. Rev Adm Pública. 2000; 34(6):35-45.

18. Trevisan LN, Junqueira LAP. Construindo o "pacto de gestão" no SUS: da descentralização tutelada à gestão em rede. Ciênc saúde coletiva. 2007; 12(4):839-902.

19. Oliveira Neto FS, Itani AF. Pactos da saúde e a gestão do SUS: experiência e avaliação na macrorregião sul do Espírito Santo. Interfacehs. 2008; 3(3):1-29.

20. Campos GW. Subjetividade e administração de pessoal: considerações sobre modos de gerenciar trabalhos em Equipes de saúde. In: Mehry EE, Onoko R (org). Agir em saúde: um desafio para um público. São Paulo: Hucitec; 1997, 29-66.

21. Associação Brasileira de Empresas de Pesquisa. Critério de classificação econômica Brasil. http://www. abep. org/Servicos/Download. aspx? id= 07. 2008 Jul 26.

22. São Paulo. Secretaria Municipal de Saúde. Diretrizes para atenção em saúde bucal. 2009.

23. Alves FNM. Organização da atenção em saúde bucal na Estratégia Saúde da Família: análise da utilização da abordagem sócio-dental e do Índice de Desenvolvimento Familiar Manguinhos/RJ [dissertação]. Rio de Janeiro: Escola Nacional de Saúde Pública da Fundação Oswaldo Cruz; 2011.

24. Roncalli AG. A organização da demanda em serviços públicos de saúde bucal: universalidade, equidade e integralidade em saúde bucal coletiva [tese]. Araçatuba: Universidade Estadual Paulista; 2000.

25. Santos SAS, Meneghin MC, Pereira AC. Análise da organização da demanda e grau de satisfação do 
profissional e usuário nas unidades de serviço público odontológico do Município de Campos dos Goytacazes/Rj/Brasil. Rev Odontol UNESP. 2007; 36(2):169-74.

26. Jesus WLA, Assis MMA. Revisão sistemática sobre o conceito de acesso nos serviços de saúde: contribuições do planejamento. Ciênc saúde coletiva. 2010; 15(1):161-70.

27. Brasil. Ministério da Saúde. Critérios Gerais para referência ao Centro de Especialidades Odontológicas. Brasília, 2009.

28. Starfield B. Coordenação da atenção: juntando tudo. In: Starfield B (org). Atenção primária: equilíbrio entre necessidades de saúde, serviços e tecnologia. Brasília: UNESCO, Ministério da Saúde, 2002. p.365-415

29. Martins RJ, Moimaz SAS, Garbin AJI, Gonçalves PRV, Garbin CAS. Relação entre a inserção da equipe de saúde bucal na Estratégia Saúde da Família e o nível de conhecimento dos agentes comunitários de saúde. Rev Bras Med Fam Comunidade. 2014; 9(33):343-9.

\section{CONFLITO DE INTERESSES}

Os autores declaram não haver conflitos de interesse.

\section{AUTOR PARA CORRESPONDÊNCIA}

Suzely Adas Saliba Moimaz

sasaliba@foa.unesp.br

Submetido em 08/05/2017

Aceito em 08/07/2017 